Pacific

Journal of

Mathematics

POSITIVE SEMIDEFINITE GERMS ON THE CONE

José F. Fernando And Jesús M. Ruiz 


\title{
POSITIVE SEMIDEFINITE GERMS ON THE CONE
}

\author{
José F. Fernando And Jesús M. Ruiz
}

\begin{abstract}
We show that any positive semidefinite analytic function germ on the cone $z^{2}=x^{2}+y^{2}$ is a sum of two squares of analytic function germs.
\end{abstract}

\section{Introduction and statement of the result.}

The problem of representing a positive semidefinite function $(=\mathrm{psd})$ as a sum of squares $(=$ sos) is a very old matter in real algebra and real geometry. Still, it is a difficult question always appealing the specialists. Concerning real analytic germs we can summarize what is known in a few statements.

Let $X$ be a irreducible real analytic set germ of dimension $d$. Any psd $f$ of $X$ is an sos of meromorphic germs (see [AnBrRz]) and the qualitative question is whether every psd is an sos of analytic germs. The quantitative question: How many squares, can refer either to meromorphic or analytic germs. We know the answer to the qualitative question in the regular case: YES for the line and the plane, NO otherwise (see [Rz1]). For singular curve germs we immediately realize that the answer is NO. For singular surfaces we have many examples where the answer is NO, and think there are very few where the answer is YES ([Rz2] $)$. For higher dimensional germs we guess the answer is NO. Concerning the quantitative matter, in the regular case the sharp bound for the number of squares of analytic germs is $d$ for $d=1,2$ (see $[\mathbf{R z 1}])$ and there are sos's of arbitrary length for $d \geq 3$ ([Ch et al $]$ ); bounds for the number of squares of meromorphic germs are 1,2 for $d=1,2$ (see $[\mathbf{R z 1}]$ ) and 8 for $d=3([\mathbf{J w}])$, (the general conjecture appears to be $2^{d-1}$, but for $d>3$ no bound is available yet). In the singular case the only systematic works concern curves $([\mathbf{O r}],[\mathbf{Q z}])$ : For sos's of meromorphic germs the bound is trivially 1 , and for analytic germs the bound is the multiplicity (a conjecture suggested by Becker and proved by Quarez, and that seems very good as there can be no uniform bound for curve germs in $\mathbb{R}^{3}$ ). The next case are surface germs. Concerning this we know that twice the multiplicity is a bound for the number of squares of meromorphic germs, and that is all. We can add that for $d \geq 4$ there are always sos's of analytic germs of arbitrary length ([Rz2] $)$, and notice the gap of knowledge for singular $X$ of dimension 3 .

With this state of affairs, the simplest possible question, putting together the quantitative and qualitative aspects is: For which surface germs any psd 
is a sum of two squares of analytic germs? This was given a first answer in [Rz1]: For very few which are in a short list of candidates. However, these candidates are difficult to handle: Only Brieskorn's surface $\left(x^{2}+y^{3}+z^{5}=0\right)$ and Whitney's umbrella $\left(x^{2}-z y^{2}=0\right)$ are known to hold the property above. In this short note we settle another candidate of the list: The cone $z^{2}=x^{2}+y^{2}$. The result is:

Theorem 1.1. Let $X: z^{2}=x^{2}+y^{2}$ be the cone singularity. Then any nonnegative analytic germ on $X$ is a sum of two squares of analytic function germs.

We develop the proof in several steps in the next section.

\section{Proof of the result.}

The ring of analytic function germs on the cone $X: z^{2}=x^{2}+y^{2}$ is the ring $A=\mathbb{R}\{x, y, z\} /\left(z^{2}-x^{2}-y^{2}\right)$, which is a free module of rank 2 over $\mathbb{R}\{x, y\}$ with basis $1, z$. This follows by division by $z^{2}-x^{2}-y^{2}$, and describes the elements in $A$ as $f(x, y)+z g(x, y) \in \mathbb{R}\{x, y\}[z]$, operations as usual with the condition that $z^{2}=x^{2}+y^{2}$. We will freely use this.

We start the proof of 1.1 by a particular case:

Proposition 2.1. Let $f, g \in \mathbb{R}[x, y]$ be such that $f+z g$ is psd on $X$. Then for some integer $m \geq 0, z^{m}(f+z g)$ is a sum of two squares of analytic germs on $X$.

Proof. First we estimate the orders of the series $f, g$. To that end, note that a planar curve germ $(a t, b t)$ lifts to two curve germs in the cone, namely $(a t, b t, \pm c t)$ with $c=\sqrt{a^{2}+b^{2}}$. Since $f+z g \geq 0$ on the cone near the origin, we get

$$
0 \leq f(a t, b t) \pm \operatorname{ctg}(a t, b t)=\left(f_{p}(a, b) t^{p}+\cdots\right) \pm\left(c g_{q}(a, b) t^{q+1}+\cdots\right),
$$

where $f_{p}$ is the initial form of $f$ and $g_{q}$ that of $g$. For a good choice of $(a, b)$ we have $f_{p}(a, b) \neq 0$ and $g_{q}(a, b) \neq 0$, and it is clear then that the above inequality implies $p \leq q+1$ and $p$ even, say $p=2 n$. Thus we can expand $f$ and $g$ into homogeneous components in the form

$$
f=f_{2 n}+\cdots+f_{r}, g=g_{2 n-1}+\cdots+g_{r-1} .
$$

In particular, the initial form of $f+z g$ is $f_{2 n}+z g_{2 n-1}$, which we can suppose does not vanish on the cone (otherwise, we replace $f$ by $f-f_{2 n}$ and $g$ by $g-g_{2 n-1}$, getting the same function on the cone).

We consider the blowing-up $\varphi: x=u t, y=v t, z=t$, which maps the cilinder $Y: u^{2}+v^{2}=1$ onto the cone. In fact, $\varphi$ maps the germ of $Y$ along the circle $t=0, u^{2}+v^{2}=1$ onto the germ of $X$ at the origin. We obtain

$$
f \circ \varphi=f(t u, t v)+t g(t u, t v)=t^{2 n} h(u, v, t),
$$


where

$$
\begin{aligned}
h(u, v, t)=\left(f_{2 n}(u, v)+g_{2 n-1}(u, v)\right)+t & \left(f_{2 n+1}(u, v)+g_{2 n}(u, v)\right)+\cdots \\
& +t^{r-2 n}\left(f_{r}(u, v)+g_{r-1}(u, v)\right) .
\end{aligned}
$$

Notice here that since $f_{2 n}(x, y)+z g_{2 n-1}(x, y)$ does not vanish on $z^{2}=x^{2}+y^{2}$, then $f_{2 n}(u, v)+g_{2 n-1}(u, v)$ does not vanish on $u^{2}+v^{2}=1$. Also, as $f+z g$ is psd on $z^{2}=x^{2}+y^{2}$ near the origin, we have $h_{t}(u, v) \geq 0$ for $u^{2}+v^{2}=1$, and $|t|$ small. From now on, our argument will be a kind of parametrized remake of the classical proof that any psd on the circle is a sum of two squares of polynomials, as it can be found in $[\mathbf{P o S z}]$. Of course, this naive presentation becomes quite involved along the way.

We consider the trigonometric polynomials for $u=\cos \theta, v=\sin \theta$ :

$$
\begin{aligned}
& \cos (k \theta)=P_{k}(u) \text { where } P_{k} \text { has degree } k, \quad \text { and } \\
& \sin (k \theta)=v Q_{k-1}(u) \text { where } Q_{k-1} \text { has degree } k-1 .
\end{aligned}
$$

Then, for $u^{2}+v^{2}=1$ we have an expression:

$$
\begin{aligned}
h(u, v, t)=\gamma_{0}(t)+\lambda_{1}(t) & P_{1}(u)+\cdots+\lambda_{r}(t) P_{r}(u) \\
& +v\left(\mu_{1}(t)+\mu_{2}(t) Q_{1}(u)+\cdots+\mu_{r}(t) Q_{r-1}(u)\right),
\end{aligned}
$$

where $\gamma_{0}, \lambda_{k}, \mu_{k} \in \mathbb{R}[t]$ have degree $\leq r-2 n$, and $\lambda_{r}, \mu_{r}$ are not both zero. On the other hand, let $\Re$ stand for real part, and $\Im$ for imaginary part (see $[\mathbf{R z} 1])$. From the relations

$$
P_{k}(u)=\Re\left((u+\sqrt{-1} v)^{k}\right), v Q_{k-1}(u)=\Im\left((u+\sqrt{-1} v)^{k}\right),
$$

we deduce

$$
\begin{aligned}
h(u, v, t)= & \gamma_{0}+\sum_{k} \lambda_{k} \Re\left((u+\sqrt{-1} v)^{k}\right)+\mu_{k} \Im\left((u+\sqrt{-1} v)^{k}\right) \\
= & \gamma_{0}+\sum_{k} \frac{1}{2}\left(\lambda_{k}+\sqrt{-1} \mu_{k}\right)(u-\sqrt{-1} v)^{k} \\
& +\frac{1}{2}\left(\lambda_{k}-\sqrt{-1} \mu_{k}\right)(u+\sqrt{-1} v)^{k} .
\end{aligned}
$$

Now, setting $w=u+\sqrt{-1} v$, we have $w \bar{w}=1$ and get

$$
\begin{aligned}
w^{r} h(u, v, t) & =w^{r}\left(\gamma_{r} \bar{w}^{r}+\cdots+\gamma_{1} \bar{w}+\gamma_{0}+\bar{\gamma}_{1} w+\cdots+\bar{\gamma}_{r} w^{r}\right) \\
& =\gamma_{r}+\cdots+\gamma_{1} w^{r-1}+\gamma_{0} w^{r}+\bar{\gamma}_{1} w^{r+1}+\cdots+\bar{\gamma}_{r} w^{2 r} \\
& =G(t, w),
\end{aligned}
$$

where $\gamma_{k}=\frac{1}{2}\left(\lambda_{k}+\sqrt{-1} \mu_{k}\right)$ for $k \geq 1$. Recall that all this holds for $u=$ $\cos \theta, v=\sin \theta$, but anyway we have this polynomial $G(t, w) \in \mathbb{C}[t, w]$. We remark here that the construction gives

$$
G(0, w)=w^{r}\left(f_{2 n}(u, v)+g_{2 n-1}(u, v)\right)
$$


for $u^{2}+v^{2}=1$, which says that $G(0, w) \neq 0$, and viewed as a series in $t$, this polynomial has order 0 . We will study $G(t, w)$ as a polynomial in $w$, whose roots are Puiseux series in the variable $t$.

First of all those roots are associated by the property that:

(1) No root of $G$ is zero, and if $\zeta$ is a root, then $1 / \bar{\zeta}$ is also a root of the same multiplicity.

Let $\zeta$ be a root of $G$. Since $G(t, 0)=\gamma_{r}=\frac{1}{2}\left(\lambda_{r}+\sqrt{-1} \mu_{r}\right)$, and $\lambda_{r}, \mu_{r}$ are not both zero, we have $\zeta \neq 0$. Moreover, we have

$$
w^{2 r} \bar{G}(t, 1 / w)=G(w),
$$

and the substitution $w=1 / \bar{\zeta}$ gives $G(t, 1 / \bar{\zeta})=0$. The assertion about the multiplicity follows from the same substitution after derivation of the above equality with respect to $w$.

We distinguish a special case for these roots, studying conjugation over the quotient field of $\mathbb{C}\{t\}$.

(2) If $\zeta$ and $1 / \bar{\zeta}$ are conjugated, then both roots have order 0 and even multiplicity, and their irreducible polynomial belongs to $\mathbb{C}\{t\}[w]$.

Interchanging $\zeta$ and $1 / \bar{\zeta}$ we can suppose $\zeta=\xi\left(t^{1 / q}\right)$ for some convergent series $\xi$. We recall that the conjugates of $\zeta=\xi\left(t^{1 / q}\right)$ are the series $\zeta^{k}=\xi\left(e^{\frac{2 \pi i}{q} k} t^{1 / q}\right), 1 \leq k \leq q$, and the irreducible polynomial of $\zeta$ is the product $\prod_{k}\left(w-\zeta^{k}\right)$ (see $\left.[\mathbf{R z} 1]\right)$, which already gives the last assertion of the statement. Now, suppose $\zeta^{k}=1 / \bar{\zeta}$. This implies that

$$
1=\xi\left(e^{\frac{2 \pi i}{q} k} t^{1 / q}\right) \bar{\xi}\left(t^{1 / q}\right)=\xi\left(e^{\frac{\pi i}{q} k} t^{1 / q}\right) \bar{\xi}\left(e^{-\frac{\pi i}{q} k} t^{1 / q}\right)
$$

(after the substitution $\left.t^{1 / q} \mapsto e^{-\frac{\pi i}{q} k} t^{1 / q}\right)$, and setting $\eta=\xi\left(e^{\frac{\pi i}{q} k} t^{1 / q}\right)$, we get $\bar{\eta}=\bar{\xi}\left(e^{-\frac{\pi i}{q} k} t^{1 / q}\right)$ and $1=\eta \bar{\eta}$. Also note that $\xi$ must have order 0 .

Let us take a closer look at the new Puiseux series $\eta$. Firstly, since $\left(e^{-\frac{\pi i}{q} k}\right)^{q}=(-1)^{k}$, the substitution $t^{1 / q} \mapsto e^{\frac{\pi i}{q} k} t^{1 / q}$ transforms the polynomial $G(t, w)$ in the polynomial $G\left((-1)^{k} t, w\right)$, and $\zeta$ in $\eta$. Thus, $\eta$ is a root of $G\left((-1)^{k} t, w\right)$ and we are reduced to show that $\eta$ has even multiplicity.

To that end, we consider a real variable $\theta$ and write:

$$
\Gamma(t, \theta)=h\left(\cos \theta, \sin \theta,(-1)^{k} t\right)=e^{-i r \theta} G\left((-1)^{k} t, e^{i \theta}\right) .
$$

For $s \in \mathbb{R}$ we have the analytic function $\Gamma_{s}: \theta \mapsto \Gamma\left((-1)^{k} s, \theta\right)$. By succesive derivations we obtain

$$
\frac{\partial^{k}}{\partial \theta^{k}} \Gamma(s, \theta)=\sum_{\ell=0}^{k} c_{k \ell} \frac{\partial^{\ell}}{\partial w^{\ell}} G\left((-1)^{k} s, e^{i \theta}\right),
$$


with $c_{k k} \neq 0$. From these formulae one sees that if $e^{i \theta} \in \mathbb{C}$ is a root of the polynomial $G_{s}=G\left((-1)^{k} s, w\right) \in \mathbb{C}[w]$, then $\theta \in \mathbb{R}$ is a root of the same multiplicity of $\Gamma_{s}$. But by the construction, the real function $\Gamma_{s}$ is $\geq 0$ for any $\theta \in \mathbb{R}$, which readily implies that all of its real roots have even multiplicity. Now, if $\eta$ has multiplicity $p$, then a suitable specialization gives a root $e^{i \theta} \in \mathbb{C}$ of multiplicity $p$ of $G_{s}$ for some $s$, and we conclude that $p$ is even.

Thus the proof of (2) is complete, and we factorize:

$$
G(t, w)=c Q^{2} \prod_{\ell}\left(w-\zeta_{\ell}\right)\left(w-\frac{1}{\bar{\zeta}_{\ell}}\right),
$$

where $c=\bar{\gamma}_{r} \in \mathbb{C}[t]$, and the factor $Q(t, w) \in \mathbb{C}\{t\}[w]$ corresponds to all roots of order 0 and even multiplicity of $G$, so that $Q$ has order 0 . Also, maybe interchanging some $\zeta_{\ell}$ and $1 / \bar{\zeta}_{\ell}$, we can suppose all $\zeta_{\ell}$ 's have order $\geq 0$. What is important is that, by $(2)$, the roots $\zeta_{\ell}$ and $1 / \bar{\zeta}_{\ell}$ are not conjugated.

After this preparation we claim:

$$
\text { The product } \prod_{\ell}\left(w-\zeta_{\ell}\right) \text { is in fact a polynomial in } \mathbb{C}\{t\}[w] \text {. }
$$

Indeed, the claim will follow from a close inspection of the conjugated roots of the $\zeta_{\ell}$ 's. Let $\zeta_{\ell}$ and $\zeta^{\prime}$ be conjugated. If $\zeta^{\prime}$ is conjugated of some $1 / \bar{\zeta}_{\ell^{\prime}}$, then $\ell^{\prime} \neq \ell$ and the order of $\zeta_{\ell^{\prime}}$ is zero, so that we can interchange $\zeta_{\ell^{\prime}}$ and $1 / \bar{\zeta}_{\ell^{\prime}}$. By this means, we guarantee that no root of $\prod_{\ell}\left(w-\zeta_{\ell}\right)$ is conjugated of a roots of $\prod_{\ell}\left(w-\frac{1}{\bar{\zeta}_{\ell}}\right)$. But by the form of our factorization, no root of $\prod_{\ell}\left(w-\zeta_{\ell}\right)$ is conjugated of a root of $Q$. Whence, we conclude that $\prod_{\ell}\left(w-\zeta_{\ell}\right)$ is a product of irreducible polynomials of $\zeta_{\ell}$ 's, all of them of order $\geq 0$, and consequently a product of polynomials in $\mathbb{C}\{t\}[w]$, so in this ring as well.

Coming back to the starting point that $h(u, v, t) \geq 0$ for $|w|=u^{2}+v^{2}=1$ and $t$ real near 0 , we can write:

$$
\begin{aligned}
h(u, v, t)=|h(u, v, t)| & =\left|w^{r} h(u, v, t)\right|=|G(t, w)| \\
& =|c|\left|Q^{2}\right| \prod_{\ell}\left|w-\zeta_{\ell}\right|\left|w-\frac{1}{\bar{\zeta}_{\ell}}\right| \\
& =\left|\frac{c}{\prod_{\ell} \bar{\zeta}_{\ell}}\right|\left|Q^{2}\right| \prod_{\ell}\left|w-\zeta_{\ell}\right|\left|\bar{\zeta}_{\ell} w-1\right| \\
& =\left|\frac{c}{\prod_{\ell} \zeta_{\ell}}\right|\left|Q^{2}\right| \prod_{\ell}\left|w-\zeta_{\ell}\right|^{2},
\end{aligned}
$$


the last equality because $\left|w-\zeta_{\ell}\right|=\left|\bar{\zeta}_{\ell} w-1\right|$ for $|w|=1$ and $t$ real. Thus we have obtained

$$
h(u, v, t)=\left|\frac{c}{\prod_{\ell} \zeta_{\ell}}\right|\left|Q \prod_{\ell}\left(w-\zeta_{\ell}\right)\right|^{2} .
$$

This formula explains itself, but we still have to look at the first factor. To that end, we analyze the product

$$
c \prod_{\ell}\left(w-\zeta_{\ell}\right)\left(w-\frac{1}{\bar{\zeta}_{\ell}}\right) \in \mathbb{C}\{t\}[w] .
$$

Setting

$$
\prod_{\ell}\left(w-\zeta_{\ell}\right)=w^{p}+\alpha_{p-1} w^{p-1}+\cdots+\alpha_{1} w+\alpha_{0},
$$

a straightforward computation shows that

$$
\prod_{\ell}\left(w-\frac{1}{\bar{\zeta}_{\ell}}\right)=w^{p}+\frac{\bar{\alpha}_{1}}{\bar{\alpha}_{0}} w^{p-1}+\cdots+\frac{\bar{\alpha}_{p-1}}{\bar{\alpha}_{0}} w+\frac{1}{\bar{\alpha}_{0}} .
$$

This will give us explicit expressions for the coefficients $\beta_{k}=\eta_{k} / \bar{\alpha}_{0}$

$$
P(t, w)=\prod_{\ell}\left(w-\zeta_{\ell}\right)\left(w-\frac{1}{\bar{\zeta}_{\ell}}\right)=w^{2 p}+\beta_{2 p-1} w^{2 p-1}+\cdots+\beta_{1} w+\beta_{0} .
$$

We recall that $G(t, w)=c Q^{2} P$ is a series of order 0 in $t$, hence the same is true for $c P$. Consequently, the order in $t$ of all the products $c \beta_{k}$ must be $\geq 0$, and some of them exactly 0 . For a few lines, let ord $d_{t}$ denote order with respect to $t$. We have:

$$
\beta_{p}=\frac{1}{\bar{\alpha}_{0}}\left(\left|\alpha_{0}\right|^{2}+\cdots+\left|\alpha_{p-1}\right|^{2}+1\right),
$$

so that $\operatorname{ord}_{t}\left(\bar{\alpha}_{0}\right) \leq \operatorname{ord}_{t}(c)$. Then

$$
0 \leq \operatorname{ord}_{t}\left(c \beta_{k}\right)=\left(\operatorname{ord}_{t}(c)-\operatorname{ord}_{t}\left(\bar{\alpha}_{0}\right)\right)+\operatorname{ord}_{t}\left(\eta_{k}\right),
$$

and this is 0 only if $\operatorname{ord}_{t}(c)=\operatorname{ord}_{t}\left(\bar{\alpha}_{0}\right)$. As $\operatorname{ord}_{t}\left(\bar{\alpha}_{0}\right)=\operatorname{ord}_{t}\left(\alpha_{0}\right)$, we deduce that $c / \alpha_{0}=c / \prod_{\ell} \zeta_{\ell}$ is a unit $a \in \mathbb{C}\{t\}$, so that $|a|^{2}$ is a unit of $\mathbb{R}\{t\}$, hence a square, say $|a|^{2}=b^{2}, b \in \mathbb{R}\{t\}$. Now, as $w=u+\sqrt{-1} v$, we can write

$$
H(u, v, t)=Q \prod_{\ell}\left(w-\zeta_{\ell}\right) \in \mathbb{C}\{t\}[u, v]
$$

in the form $H=H_{1}+\sqrt{-1} H_{2}, H_{1}, H_{2} \in \mathbb{R}\{t\}[u, v]$, and $|H|^{2}=H_{1}^{2}+H_{2}^{2}$. The conclusion is that

$$
h(u, v, t)=b(t)^{2}\left(H_{1}(u, v, t)^{2}+H_{2}(u, v, t)^{2}\right)
$$


all this for $u^{2}+v^{2}=1, t$ small. Hence we change $u=x / z, v=y / z, t=z$, to find

$$
\begin{aligned}
f(x, y)+z g(x, y) & =t^{2 n} h(u, v, t) \\
& =z^{2 n} b(z)^{2}\left(H_{1}(x / z, y / z, t)^{2}+H_{2}(x / z, y / z, t)^{2}\right),
\end{aligned}
$$

and finally note that $H_{1}$ and $H_{2}$ being polynomials in the variables $u, v$, multiplying by a large power $m$ of $z$, we get $z^{m} H_{1}(x / z, y / z, t), z^{m} H_{2}(x / z, y / z, t)$ $\in \mathbb{R}\{t\}[x, y]$, and so

$$
\begin{aligned}
z^{2 m}(f(x, y)+z g(x, y))= & z^{2 n} b(z)^{2}\left(\left(z^{m} H_{1}(x / z, y / z, t)\right)^{2}\right. \\
& \left.+\left(z^{m} H_{2}(x / z, y / z, t)\right)^{2}\right)
\end{aligned}
$$

is a sum of two squares of analytic functions on the cone $z^{2}=x^{2}+y^{2}$.

The next step is to get rid of the denominator $z^{2 m}$ in the preceding proposition. For this we prove:

Proposition 2.2. Suppose that the function $f(x, y)+z g(x, y)$ has the property that $z^{2 m}(f+z g)$ is a sum of two squares of analytic germs on the cone. Then $f+z g$ itself is a sum of two squares of analytic germs.

Proof. By hypothesis, there are series $a_{1}, a_{2}, b_{1}, b_{2} \in \mathbb{R}\{x, y\}$ and $h \in \mathbb{R}\{x$, $y, z\}$ such that

$$
\left(x^{2}+y^{2}\right)^{m}(f+z g)=\left(a_{1}+z b_{1}\right)^{2}+\left(a_{2}+z b_{2}\right)^{2}-\left(z^{2}-x^{2}-y^{2}\right) h .
$$

By the Weierstrass Division Theorem (see $[\mathbf{R z} 1]$ ), $h$ must be a polynomial in $z$, and looking at the degrees in both sides we conclude that in fact $h \in \mathbb{R}\{x, y\}$, and in the end

$$
\begin{gathered}
h=b_{1}^{2}+b_{2}^{2}, \quad\left(x^{2}+y^{2}\right)^{m} g=2 a_{1} b_{1}+2 a_{2} b_{2}, \\
\left(x^{2}+y^{2}\right)^{m} f-\left(x^{2}+y^{2}\right) h=a_{1}^{2}+a_{2}^{2} .
\end{gathered}
$$

Now we compute a little:

$$
\begin{aligned}
\left(\left(x^{2}+y^{2}\right)^{m} f-\left(x^{2}+y^{2}\right) h\right) h & =\left(a_{1}^{2}+a_{2}^{2}\right)\left(b_{1}^{2}+b_{2}^{2}\right) \\
& =\left(a_{1} b_{1}+a_{2} b_{2}\right)^{2}+\left(a_{1} b_{2}-a_{2} b_{1}\right)^{2} \\
& =\frac{1}{4}\left(x^{2}+y^{2}\right)^{2 m} g^{2}+\left(a_{1} b_{2}-a_{2} b_{1}\right)^{2},
\end{aligned}
$$

so that $x^{2}+y^{2}$ divides $a_{1} b_{2}-a_{2} b_{1}$. Since it also divides $a_{1} b_{1}+a_{2} b_{2}$, we deduce it divides

$$
\begin{gathered}
\left(a_{1} b_{2}-a_{2} b_{1}\right) b_{2}+\left(a_{1} b_{1}+a_{2} b_{2}\right) b_{1}=a_{1}\left(b_{1}^{2}+b_{2}^{2}\right)=a_{1} h \\
-\left(a_{1} b_{2}-a_{2} b_{1}\right) b_{1}+\left(a_{1} b_{1}+a_{2} b_{2}\right) b_{2}=a_{2}\left(b_{1}^{2}+b_{2}^{2}\right)=a_{2} h .
\end{gathered}
$$

Suppose first $m \geq 2$, and that $x^{2}+y^{2}$ does not divide $h$. Then it divides both $a_{1}$ and $a_{2}$, hence $\left(x^{2}+y^{2}\right)^{2}$ divides $a_{1}^{2}+a_{2}^{2}=\left(x^{2}+y^{2}\right)^{m} f-\left(x^{2}+y^{2}\right) h$, 
and $x^{2}+y^{2}$ divides $h$, a contradiction. Thus $x^{2}+y^{2}$ divides $h$, so that it divides $\left(a_{1}+z b_{1}\right)^{2}+\left(a_{2}+z b_{2}\right)^{2}=\alpha_{1}^{2}+\alpha_{2}^{2}$. Factorizing in $\mathbb{C}\{x, y, z\}$ we find that $x+\sqrt{-1} y$ divides $\alpha_{1}+\sqrt{-1} \alpha_{2}$ (or the conjugate, but then replace $\alpha_{2}$ by $\left.-\alpha_{2}\right)$. We write this:

$$
\begin{aligned}
& \alpha_{1}+\sqrt{-1} \alpha_{2}=(x+\sqrt{-1} y)\left(\beta_{1}+\sqrt{-1} \beta_{2}\right), \\
& \alpha_{1}-\sqrt{-1} \alpha_{2}=(x-\sqrt{-1} y)\left(\beta_{1}-\sqrt{-1} \beta_{2}\right),
\end{aligned}
$$

where $\beta_{1}, \beta_{2} \in \mathbb{R}\{x, y, z\}$. Whence on the cone $z^{2}=x^{2}+y^{2}$ we have

$$
\left(x^{2}+y^{2}\right)^{m}(f+z g)=\alpha_{1}^{2}+\alpha_{2}^{2}=\left(x^{2}+y^{2}\right)\left(\beta_{1}^{2}+\beta_{2}^{2}\right),
$$

and simplifying $x^{2}+y^{2}$ we see that $\left(x^{2}+y^{2}\right)^{m-1}(f+z g)$ is a sum of two squares of analytic germs.

Repeating the argument above we end up with $f+z g$ being a sum of two squares of analytic germs or with $m=1$ and $x^{2}+y^{2}$ dividing both $a_{1}$ and $a_{2}$. If that is the case, we can write $a_{1}=\left(x^{2}+y^{2}\right) a_{1}^{\prime}, a_{2}=\left(x^{2}+y^{2}\right) a_{2}^{\prime}$, and on the cone

$$
\begin{aligned}
z^{2}(f+z g)=\left(a_{1}+z b_{1}\right)^{2}+\left(a_{2}+z b_{2}\right)^{2} & =\left(z^{2} a_{1}^{\prime}+z b_{1}\right)^{2}+\left(z^{2} a_{2}^{\prime}+z b_{2}\right)^{2} \\
& =z^{2}\left(\left(z a_{1}^{\prime}+b_{1}\right)^{2}+\left(z a_{2}^{\prime}+b_{2}\right)^{2}\right),
\end{aligned}
$$

so that in fact $f+z g$ is a sum of two squares of analytic functions.

Combining the two preceding results, we have solved the problem for functions $f+z g$ where $f, g$ are polynomials. For the general case we only need the following approximation lemma:

Lemma 2.3. Let $H$ be a non-negative function germ on the cone. Then, for every $k \geq 0$ there is a non-negative function germ on the cone $f+z g$, with $f, g$ polynomials, such that $H \equiv f+z g \bmod (x, y, z)^{k}$.

For, let us assume this. What we must show is that the equation

$$
H=\mathrm{h}_{1}^{2}+\mathrm{h}_{2}^{2}+\left(z^{2}-x^{2}-y^{2}\right) \mathrm{h}
$$

has a solution $\mathrm{h}_{1}=h_{1}, \mathrm{~h}_{2}=h_{2}, \mathrm{~h}=h$ in $\mathbb{R}\{x, y, z\}$. By M. Artin's Approximation Theorem (see $[\mathbf{R z} 1]$ and $[\mathbf{B b}$ et $\mathbf{a l}]$ ), a solution exists if it exists up to order $k$ large enough, that is, if the congruence

$$
H \equiv \mathrm{h}_{1}^{2}+\mathrm{h}_{2}^{2}+\left(z^{2}-x^{2}-y^{2}\right) \mathrm{h} \quad \bmod (x, y, z)^{k}
$$

has a solution for $k$ large. But the psd $f+z g$ provided by 2.3 is a sum of two squares by the polynomial case 2.1: There are $h_{1}, h_{2}, h \in \mathbb{R}\{x, y, z\}$ such that $f+z g=h_{1}^{2}+h_{2}^{2}+\left(z^{2}-x^{2}-y^{2}\right) h$. Clearly $h_{1}, h_{2}, h$ are the solution up to order $k$ we sought, and we are done.

To finish, let us prove the lemma. 
Proof of 2.3. First, replacing $H$ by $\left(x^{2}+y^{2}\right)^{k}+H$ we can assume that $H$ vanishes only at the origin. Then we write $H=f+z g$ with $f, g \in \mathbb{R}\{x, y\}$. If $f=0$ contains some planar curve germ $Y$, this germ $Y$ lifts to two curve germs $Y_{+}$and $Y_{-}$in the cone corresponding to $z= \pm \sqrt{x^{2}+y^{2}}$. On these curve germs $H=z g \geq 0$, and, $z$ changing sign from $Y_{+}$to $Y_{-}$, this is only possible if $g$ vanishes on $Y$, and then $H$ vanishes on $Y_{+}$and $Y_{-}$, against the assumption that it only vanishes at the origin. All this means that $f$ only vanishes at the origin. Next we use again the trick that the change $z=-z$ leaves the cone invariant, so that both $f+z g$ and $f-z g$ vanish only at the origin, hence so does their product $f^{2}-z^{2} g^{2}=f^{2}-\left(x^{2}+y^{2}\right) g^{2} \in \mathbb{R}\{x, y\}$. This means that all the complex branches of $f$ and $f^{2}-\left(x^{2}+y^{2}\right) g^{2}$ are imaginary. Now, we recall an easy consequence of the Newton algorithm (see $[\mathbf{W k}]$ ) for the computation of the complex branches of a planar curve germ: The jets of order $r$ of those branches depend on the jets of order $s$ of the given germ, or in other words, if we approximate the curve germ till order $s$, the branches of the approximation approximate the branches of the given germ till order $r$. Hence if we have a germ whose branches are all imaginary, any close approximation of the germ has also this property. Applying this to $f$ and $f^{2}-\left(x^{2}+y^{2}\right) g^{2}$ we find that replacing $f$ and $g$ by their jets of order high enough (and $\geq k), f$ and $f^{2}-\left(x^{2}+y^{2}\right) g^{2}$ have only imaginary branches, and are $\geq 0$. Consequently, after the replacement by those jets we still have

$$
\begin{gathered}
(f+z g)+(f-z g)=2 f \geq 0, \\
(f+z g)(f-z g)=f^{2}-\left(x^{2}+y^{2}\right) g^{2} \geq 0 .
\end{gathered}
$$

Whence, $f+z g \geq 0$, and we are done.

\section{References}

[AnBrRz] C. Andradas, L. Bröcker and J.M. Ruiz, Constructible Sets in Real Geometry, Ergeb. Math., 33, Springer Verlag, Berlin-Heidelberg-New York, 1996, MR 98e:14056, Zbl 0873.14044.

$[\mathrm{Bb}$ et al] S. Basarab, V. Nica and D. Popescu, Approximation properties and existencial completeness for ring morphisms, Manuscripta Math., 33 (1981), 227-282, MR 82k:03047, Zbl 0472.13013.

[Ch et al] M.D. Choi, Z.D. Dai, T.Y. Lam and B. Reznick, The Pythagoras number of some affine algebras and local algebras, J. Reine Angew. Math., 336 (1982), 45-82, MR 84f:12012, Zbl 0523.14020.

[Jw] P. Jaworski, About estimates on mumber of squares necessary to represent a positive-semidefinite analytic function, Arch. Math., 58 (1992), 276-279, MR 93c:32008, Zbl 0748.14021.

[Or] J. Ortega, On the Pythagoras number of a real irreducible algebroid curve, Math. Ann., 289 (1991), 111-123, MR 92a:14065, Zbl 0743.14041. 
[PoSz] G. Pólya and G. Szegö, Problems and Theorems in Analysis I \& II, Springer Study Edition, Springer Verlag, New York-Heidelberg-Berlin, 1976, MR 49 \#8782, MR 53 \#2.

[Qz] R. Quarez, Pythagoras numbers of real algebroid curves and Gram matrices, J. Algebra, 238(1) (2001), 139-158.

[Rz1] J.M. Ruiz, The Basic Theory of Power Series, Advanced Lectures in Mathematics, Vieweg Verlag, Braunschweig Wiesbaden, 1993, MR 94i:13012.

[Rz2] Sums of two squares in analytic rings, Math. Z., 230 (1999), 317-328, Zbl 0930.32007.

[Wk] R.J. Walker, Algebraic Curves, Springer Verlag, Berlin-Heidelberg-New York, 1996, MR 80c:14001, Zbl 0399.14016.

Received July 19, 2000 and revised November 22, 2000.

Depto. Algebra

Depto. Geometría y Topología

F. Ciencias Matemáticas

Universidad Complutense de Madrid

28040 MADRID

SPAIN

E-mail address: josefer@mat.ucm.es

Depto. Algebra

Depto. Geometría y Topología

F. Ciencias matemáticas

Universidad Complutense de Madrid

28040 MADRID

SPAIN

E-mail address: jesusr@mat.sim.ucm.es 